\title{
A Method for Reconstruction of Boiler Combustion Temperature Field Based on Acoustic Tomography
}

\author{
Yuhui Wu, ${ }^{1}$ Xinzhi Zhou ${ }^{10},{ }^{1}$ Li Zhao, ${ }^{1}$ Chenlong Dong, ${ }^{2}$ and Hailin Wang ${ }^{1}$ \\ ${ }^{1}$ School of Electronics and Information Engineering, Sichuan University, Chengdu 610065, China \\ ${ }^{2}$ Nuclear Power Institute of China, Chengdu 610200, China \\ Correspondence should be addressed to Xinzhi Zhou; xz.zhou@scu.edu.cn
}

Received 2 April 2021; Revised 26 July 2021; Accepted 17 August 2021; Published 2 September 2021

Academic Editor: Babak Shotorban

Copyright (c) 2021 Yuhui Wu et al. This is an open access article distributed under the Creative Commons Attribution License, which permits unrestricted use, distribution, and reproduction in any medium, provided the original work is properly cited.

Acoustic tomography (AT), as a noninvasive temperature measurement method, can achieve temperature field measurement in harsh environments. In order to achieve the measurement of the temperature distribution in the furnace and improve the accuracy of AT reconstruction, a temperature field reconstruction algorithm based on the radial basis function (RBF) interpolation method optimized by the evaluation function (EF-RBFI for short) is proposed. Based on a small amount of temperature data obtained by the least square method (LSM), the RBF is used for interpolation. And, the functional relationship between the parameter of RBF and the root-mean-square (RMS) error of the reconstruction results is established in this paper, which serves as the objective function for the effect evaluation, so as to determine the optimal parameter of RBF. The detailed temperature description of the entire measured temperature field is finally established. Through the reconstruction of three different types of temperature fields provided by Dongfang Boiler Works, the results and error analysis show that the EF-RBFI algorithm can describe the temperature distribution information of the measured combustion area globally and is able to reconstruct the temperature field with high precision.

\section{Introduction}

As temperature is spatially distributed and changes with time, it is necessary to measure and analyze the temperature distribution to accurately acquire comprehensive temperature information of the target area, whether in the industrial production or in the detection of people's living environment parameters. Acoustic tomography (AT), as a nonintrusive temperature measurement technique, has the advantages of wide measuring range, short measuring time delay, and good environment adaptability. Therefore, it is widely applied in temperature measurement for boiler hearth [1-5], gas [6-8], granary [9], hydrothermal solution [10-12], and noninvasive thermometry for materials [13-16].

During the operation of the boiler, the temperature distribution in the furnace directly affects the combustion efficiency of pulverized coal and the combustion safety of the boiler. It is an important parameter reflecting the combustion process and the evolution of emissions [1, 2]. The analysis of the furnace temperature field can guide the adjustment of the fuel mixing ratio, which is conducive to improving the combustion efficiency, reducing the emission of pollutants, and optimizing the combustion state of the boiler. Since the combustion process of boilers is characterized by harsh environment, complex and variable state, and local high temperature $[1,3]$, it is difficult to obtain an accurate temperature field in the furnace with traditional contact temperature measurement. With the reconstruction algorithm for temperature distribution based on AT, which is a nonintrusive temperature measurement technique, the temperature distribution of the measure area can be obtained by measuring the time-of-flight (TOF) of multiple ultrasonic propagation paths in the measure area. Currently, iterative methods, truncated singular value decomposition method (TSVD), algorithm based on RBF neural network, and least square method (LSM) are commonly used to reconstruct the temperature distribution by AT. Iterative 
methods mainly involve algebraic reconstruction technique (ART) $[1,17]$ and simultaneous algebraic reconstruction technique (SART) $[18,19]$. Due to repeated iterations, the convergence of the iterative method can be good but slow. And, the reconstruction results are easily disturbed by noises. Compared with ART, SART can reduce noise interference and then gains better robustness. TSVD [20] directly truncates the smaller singular values that make the solution unstable, but how big the singular value should be truncated is a difficult problem. The algorithm based on the RBF neural network [21, 22] has the optimal functional approximation capability at arbitrary precision. However, numerous reliable training samples, which are difficult to acquire in practice, are required to determine the appropriate parameters of the neural network. This requirement leads to a significant limitation on the practicability of this method. The LSM $[12,23]$ is commonly applied in acoustic thermometry since it is simple with high stability and can gain relatively high reconstruction accuracy. However, information loss will occur at the edges of the temperature field in the LSM. Using the Kriging model with both local and global statistical characteristics, Yan et al. [24] used the Kriging method to interpolate and extrapolate a small number of pixels after reconstructing them with LSM to get the temperature description of the whole area. Using the good ability of multiquadric interpolation to process sparse data, Shen et al. [25] proposed a reconstruction algorithm based on LSM and multiquadric interpolation so that the reconstruction results will not lose information at the edge of the area. However, how to improve the accuracy of temperature field reconstruction and the quality of reconstruction remains an open problem.

In order to improve the accuracy and quality of temperature distribution reconstruction based on AT, a new algorithm for temperature field reconstruction based on the radial basis function (RBF) interpolation method optimized by the evaluation function (EF-RBFI for short) is proposed. RBF has a strong fitting ability, and the determination of the parameter of RBF significantly affects the temperature distribution reconstruction results, as the parameter influences the smoothness of the function shape [26] which is closely related with the reconstruction results. Therefore, the proposed algorithm uses RBF to interpolate a small amount of temperature data obtained by LSM and establishes the functional relationship between the parameters of RBF and the root-mean-square (RMS) error of reconstruction results, which is used as the objective function of effect evaluation to determine the optimal parameter of RBF and the corresponding minimum RMS error of reconstruction results, so as to achieve high-precision reconstruction of the boiler combustion temperature field.

This paper is organized as follows. In Section 2, the principle of acoustic thermometry and reconstruction is introduced. Section 3 presents a reconstruction algorithm based on the RBF interpolation method optimized by the evaluation function. In Section 4, simulation experiments are conducted to evaluate the proposed algorithm and results analysis is shown. The conclusions and future research are provided in Section 5.

\section{The Principle of Acoustic Thermometry and Reconstruction}

The temperature thermometry by AT is based on the medium temperature dependence of ultrasonic velocity. The ultrasonic velocity is proportional to the square root of medium temperature, which can be expressed as follows [7]:

$$
v=\sqrt{\frac{\gamma R}{M} T}=P \sqrt{T},
$$

where $v$ represents the ultrasonic velocity in the gas $(\mathrm{m} / \mathrm{s}), \gamma$ represents the adiabatic exponent of the gas, which is related with the composition and state of the gas medium, $R$ is the universal gas constant $(\mathrm{J} /(\mathrm{mol} \cdot \mathrm{K})), M$ is the average molecular weight of the gas $(\mathrm{kg} / \mathrm{mol})$, and $T$ represents the thermodynamic temperature of gas $(\mathrm{K})$. For a certain gas medium, $M, R$, and $\gamma$ are known quantities; then, $P$ is a fixed constant.

Therefore, when the gas medium is determined and the ultrasonic velocity $v$ is known, $T$ can be expressed as

$$
T=\left(\frac{v}{P}\right)^{2} \text {. }
$$

As the distance between the ultrasonic transmitter and ultrasonic receiver (or collectively called ultrasonic transceivers) is known, the average ultrasonic velocity along the propagation path can be calculated when the TOF of the propagation path is measured. Then, the average temperature $T$ of the path can be presented as follows:

$$
T=\left(\frac{L}{t}\right)^{2} \frac{1}{P^{2}}
$$

where $L$ is the path length between the ultrasonic transceivers and $t$ is the TOF of the ultrasonic propagation path.

Thus, to obtain the temperature distribution reconstruction by AT, multiple ultrasonic transceivers are installed over the measure area. Then, the TOF of multiple ultrasonic propagation paths can be combined to compute ultrasonic velocity distribution with suitable algorithms. Finally, the temperature distribution of the measure area can be obtained by the relationship between ultrasonic velocity and temperature.

\section{Principle of Temperature Field Reconstruction Algorithm}

3.1. Characterization and Calculation of Temperature Matrix. Theoretically, the TOF of the ultrasonic signals along specific paths from ultrasonic transmitters to receivers can be expressed as

$$
t=\int \frac{1}{v} \mathrm{~d} s=\int a \mathrm{~d} s,
$$

where $t$ is the TOF of the ultrasound, $v$ is the ultrasonic velocity, $a$ represents the reciprocal of the ultrasonic velocity, and $s$ is the equation of the acoustic propagation path.

Obtain the TOF of multiple ultrasonic propagation paths through the ultrasonic velocity distribution or temperature 
distribution information in a certain area, which is a solution to a positive problem. However, the AT-based temperature field reconstruction is to obtain the ultrasonic velocity distribution or temperature distribution by measuring the TOF data, which is a solution to the inverse problem. The solution process is often ill-conditioned, and the matrix equation can be solved by the LSM method.

In the temperature distribution reconstruction, the measure area is divided into $N$ unit blocks. And, due to the limitation of LSM, $N$ should be less than the number of propagation paths $M$ [25]. Assuming that the ultrasonic velocity is evenly distributed in each unit block, then from equation (4), the corresponding TOF of ultrasound in $k$ th selected propagation path can be rewritten as

$$
t_{k}=\sum_{i=1}^{N} \Delta S_{k i} a_{i}
$$

where $a_{i}$ represents the reciprocal of the average ultrasonic velocity in the $i$ th unit block and $\Delta S_{k i}$ represents the length of the $i$ th unit block through which the $k$ th selected path passes.

Then, the difference between the actual measured value $\widehat{t}_{k}$ and the theoretical value $t_{k}$ of the ultrasonic TOF along the $k$ th selected propagation path is presented as

$$
\varepsilon_{k}=\widehat{t}_{k}-t_{k}=\widehat{t}_{k}-\sum_{i=1}^{N} \Delta S_{k i} a_{i} .
$$

The following equation is made tenable by using LSM:

$$
\frac{\partial}{\partial a_{i}} \sum_{k=1}^{M}\left(\widehat{t}_{k}-\sum_{i=1}^{N} \Delta S_{k i} a_{i}\right)^{2}=0 .
$$

Then, the canonical equation can be obtained as

$$
S^{T} \cdot S \cdot A=S^{T} \cdot t
$$

where $A=\left[a_{1} a_{2}, \ldots, a_{N}\right]^{T}, \quad t=\left[\widehat{t}_{1} \widehat{t}_{2}, \ldots, \widehat{t}_{M}\right]^{T}$, and $S=\left[\begin{array}{cccc}\Delta S_{11} & \Delta S_{12} & \cdots & \Delta S_{1 N} \\ \Delta S_{21} & \Delta S_{22} & \cdots & \Delta S_{2 N} \\ \cdots & \cdots & \cdots & \cdots \\ \Delta S_{M 1} & \Delta S_{M 2} & \cdots & \Delta S_{M N}\end{array}\right]$.

From equation (8), the reciprocal of average ultrasonic velocity of each unit block can be computed as

$$
A=\left(S^{T} S\right)^{-1} S^{T} t
$$

According to equation (2), the average temperature of each unit block can be obtained that

$$
T=\frac{1}{A^{2} P^{2}}
$$

Thus, $T=\left[T_{1} T_{2}, \ldots, T_{N}\right]^{T}$. And, it is the matrix composed of the average temperature of $N$ unit blocks.

3.2. Reconstruction Based on RBF Interpolation. The temperature distribution function of the measure area is presented by $T(x, y)$, which then is expressed as a linear combination of $N$ RBFs, as follows:

$$
T(x, y)=\sum_{i=1}^{N} \alpha_{i} r_{i}(x, y),
$$

where $\alpha_{i}$ is the undetermined coefficient and $r_{i}(x, y)$ is the inverse multiquadric radial basis function (IMQ-RBF). The expression is as follows:

$$
r_{i}(x, y)=\frac{1}{\sqrt{\left(x-x_{i}\right)^{2}+\left(y-y_{i}\right)^{2}+c^{2}}},
$$

where $c$ is the parameter of the IMQ-RBF and $\left(x_{i}, y_{i}\right)$ is the coordinate of the center point in the $i$ th unit block. The average temperature of each unit block then is regarded as its center point temperature, and they are taken as the sampling data for interpolation. The temperature matrix $T$ derived from equation (10) is substituted into equation (11), obtaining the following equation:

$$
\left\{\begin{array}{l}
T_{1}=\sum_{i=1}^{N} \alpha_{i} r_{i}\left(x_{1}, y_{1}\right), \\
T_{2}=\sum_{i=1}^{N} \alpha_{i} r_{i}\left(x_{2}, y_{2}\right), \\
\ldots \\
T_{N}=\sum_{i=1}^{N} \alpha_{i} r_{i}\left(x_{N}, y_{N}\right),
\end{array}\right.
$$

which can be rewritten in the matrix form as

$$
T=\alpha \cdot R \text {, }
$$

where $\alpha=\left[\alpha_{1} \alpha_{2}, \ldots, \alpha_{N}\right]^{T}$ represents the matrix of the undetermined coefficient and $R$ represents the matrix of function value of the RBF at the centers of $n$ unit blocks. And, $R$ is expressed as follows:

$$
R=\left[\begin{array}{cccc}
r_{1}\left(x_{1}, y_{1}\right) & r_{2}\left(x_{1}, y_{1}\right) & \cdots & r_{N}\left(x_{1}, y_{1}\right) \\
r_{1}\left(x_{2}, y_{2}\right) & r_{2}\left(x_{2}, y_{2}\right) & \cdots & r_{N}\left(x_{2}, y_{2}\right) \\
\vdots & \vdots & \ddots & \vdots \\
r_{1}\left(x_{N}, y_{N}\right) & r_{2}\left(x_{N}, y_{N}\right) & \cdots & r_{N}\left(x_{N}, y_{N}\right)
\end{array}\right] .
$$

Then, the undetermined coefficient matrix can be obtained by the following equation:

$$
\alpha=R^{-1} \cdot T .
$$

Finally, the temperature distribution of the measure area can be obtained by substituting $\alpha$ into equation (11).

\subsection{An Optimal Parameter Determination Method for RBF} Based on Evaluation Function. Since the parameter of RBF affects the smoothness and the specific shape of the function, it is also named as "shape parameter." For instance, when the parameter $c$ is $1,3,5$, and 7 , the shapes of IMQ-RBF are as shown in Figure 1.

Since the temperature distribution function is expressed by the linear combination of RBFs, the reconstructed 


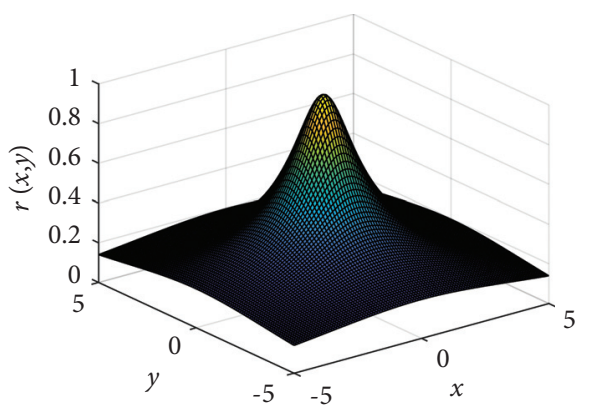

(a)

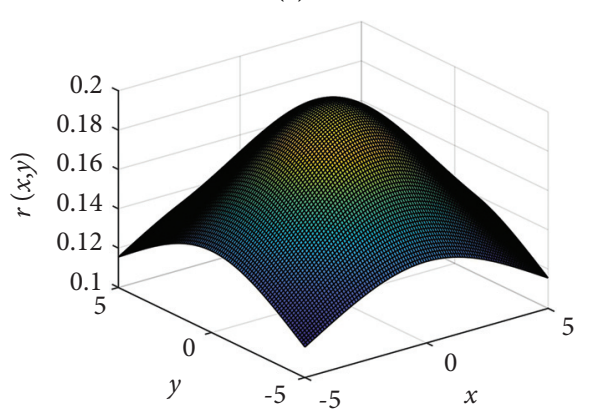

(c)

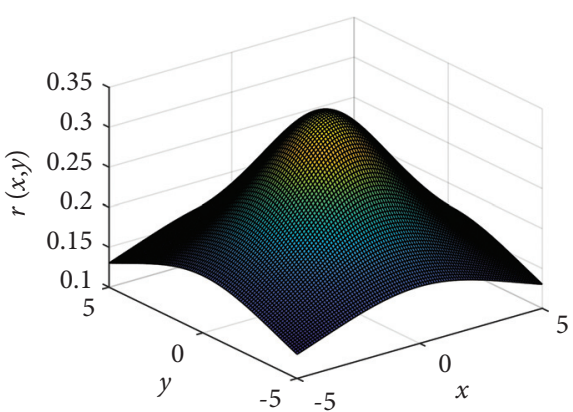

(b)

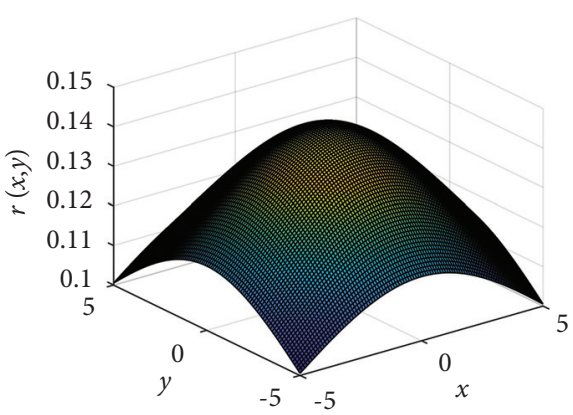

(d)

FIgURE 1: The shapes of IMQ-RBF under different parameters. (a) $c=1$. (b) $c=3$. (c) $c=5$. (d) $c=7$.

temperature distribution will change when the specific shape of RBF varies with the parameter. Consequently, the determination for the parameter of RBF is of vital importance, yet there is no efficient method to realize it. In the commonly used numerical experiment method [25,27], the parameter of RBF is manually determined through multiple attempts accompanied with comparison of the reconstruction error, which is inefficient and cannot obtain the best result.

Generally, the RMS error of reconstruction results is used to evaluate the reconstruction performance. Therefore, this paper takes RMS error of reconstruction results as the evaluation criterion and establishes the functional relationship between the parameter of RBF and the RMS error. The function is shown in equation (17), in which the evaluation function should be minimized to obtain the optimal parameter of RBF:

$$
f(c)=\frac{\sqrt{1 / n \sum_{i=1}^{n}\left(\mathrm{TR}_{i}(c)-\mathrm{TM}_{i}\right)^{2}}}{\mathrm{TM}_{\text {mean }}},
$$

where $f(c)$ is named the evaluation function, $c$ is the parameter of RBF, $n$ is the number of divided blocks, $\mathrm{TR}_{i}$ and $\mathrm{TM}_{i}$ represent the temperature value of the reconstructed temperature distribution and the measure model under the same coordinate, respectively, and $\mathrm{TM}_{\text {mean }}$ represents the average temperature of the temperature distribution model.

The optimal parameter of RBF can be determined through repetitive iterative computations searching for the parameter which minimizes the reconstruction error. Therefore, in this paper, the intelligent optimization algorithm is combined with evaluation function to efficiently determine the optimal parameter of RBF. This method can avoid contingencies and error caused by manual operation in the numerical experiment.

The differential evolution (DE) algorithm $[28,29]$ is a population-based heuristic random searching algorithm, which can intelligently generate optimization searching through cooperation and competition among individuals in the population. Compared with other optimization algorithms, the DE algorithm has the advantages of fewer parameters, better stability, and global optimization ability [30]. Meanwhile, to prevent premature convergence and to enhance the convergence speed and robustness of the DE algorithm, the mutation factor $F$ and cross factor CR are automatically adjusted in the evolution of the algorithm. This automatic adjustment enables a favorable global search ability in the early iteration stage, simultaneously guaranteeing the computation accuracy and convergence speed in the late iteration. The adaptive mutation factor $F$ and cross factor CR are expressed as follows [29]:

$$
\begin{aligned}
F & =\left(F_{\max }-F_{\text {min }}\right) \times e^{1-G /(G-g+1)}+F_{\text {min }}, \\
\mathrm{CR} & =\mathrm{CR}_{\max }-\frac{\left(\mathrm{CR}_{\max }-\mathrm{CR}_{\min }\right) g}{G},
\end{aligned}
$$

where $g$ represents the current number of iterations, $G$ is the maximum number of iterations, $F_{\max }$ and $F_{\min }$ are the maximum value and the minimum value of the mutation factor, respectively, and $\mathrm{CR}_{\max }$ and $\mathrm{CR}_{\min }$ are the maximum 
value and the minimum value of the crossover factor, respectively.

The proposed method for determining the optimal parameter of RBF in temperature distribution reconstruction is as follows. First, the temperature matrix $T$ is obtained by the reconstruction algorithm based on LSM. Next, the parameters such as population size, maximum number of iterations, mutation factor $F_{\max }$ and $F_{\min }$, and cross factor $\mathrm{CR}_{\max }$ and $\mathrm{CR}_{\min }$ are initialized in the $\mathrm{DE}$ algorithm, and the constraint range of the parameter of RBF is determined by the numerical experiment. Third, the randomly generated initial population is evaluated and the fitness of each individual in it is calculated. The calculation process of the fitness is shown in Figure 2, in which the evaluation function is taken as the objective function for computing fitness. Fourth, two distinctive individual vectors are randomly selected from the initial population to make a subtraction. And, a mutation individual can be calculated by adding a third randomly selected individual vector to the subtraction result according to certain rules, namely, to perform mutation operation. Then, the mutation individuals and the target individuals of the parent generation are crossed according to certain rules to generate a new experimental individual, namely, to perform the crossover operation. After that, the experimental individual will replace the parent individual if the fitness value of the former is better than that of the later; otherwise, the parent individual will be retained, namely, to perform the selection operation [30]. The superior individual retained in the repetitive iterations will eventually lead the searching process to reach the optimal parameter of RBF. The work flow of the method for determining the optimal parameter of RBF is shown in Figure 2.

\section{Simulation Experiments and Results' Analysis}

4.1. Design of Temperature Distribution Reconstruction and Simulation of Parameter Optimization for RBF. In order to evaluate the feasibility and effectiveness of the proposed algorithm, the EF-RBFI algorithm is used to reconstruct three kinds of furnace temperature distributions provided by Dongfang Boiler Works.

The size of the temperature field to be measured is $25 \mathrm{~m} \times 8.22 \mathrm{~m}$, which is the actual length and width of the temperature measurement area in the boiler. When reconstructing the temperature field, the arrangement of transducers is generally distributed around the area to be measured, and the principle of symmetry is satisfied as far as possible. At the same time, the ultrasonic propagation path is scattered through the area to be measured as far as possible. The number of unit blocks divided in the measured area should be appropriate. The least square method requires the number of unit blocks to be smaller than the number of ultrasonic propagation paths. If the number of unit blocks is too small, the temperature information obtained according to the reconstruction algorithm will be less. Even if interpolation processing is performed on this basis, the error of the reconstruction result obtained will be larger. If the number of unit blocks is too much, the inverse problem of reconstructing the temperature field based on AT will be seriously ill-conditioned, making the solution process extremely difficult. At the same time, the dividing line of the divided unit blocks should also be avoided to coincide with the ultrasonic propagation path; otherwise, the temperature field will not be reconstructed. As shown in Figure 3, the measure area is divided into $5 \times 3$ blocks by the dotted lines, where 10 ultrasonic transceivers (i.e., T1 to T10 in Figure 3) arranged on its four sides, and the red solid lines represent the ultrasonic propagation paths. The three temperature field distributions provided by Dongfang Boiler Works are shown in Figures 4-6(a), which are single-peak temperature field, double-peak temperature field, and high-low peak temperature field.

In the simulation, the population size is set as 20 , the maximum number of iterations is 50 , the mutation factor $F_{\max }$ and $F_{\min }$ are set as 0.9 and 0.2 , respectively, the cross factor $\mathrm{CR}_{\max }$ and $\mathrm{CR}_{\min }$ are 0.6 and 0.1 , respectively, $\mathrm{DE} /$ rand/1 is selected as the mutation strategy, binomial crossover is selected as the crossover strategy, and the evaluation function established in equation (17) is taken as the objective function for calculating the fitness. The reconstruction results of the EF-RBFI algorithm are compared with the following algorithms: the algorithm based on LSM and bicubic interpolation (LSM-BI for short), the algorithm based on LSM and cubic spline interpolation (LSM-SP for short), the algorithm based on ART and cubic spline interpolation (ART-SP for short), and the algorithm based on LSM and Kriging interpolation (LSM-KR for short).

4.2. Simulation Results and Error Analysis. Figures 4(b)-4(f), $5(\mathrm{~b})-5(\mathrm{f})$, and 6(b)-6(f) show the reconstruction results of three temperature fields implemented by different algorithms. At the same time, in order to quantitatively evaluate the temperature field reconstruction effect of various algorithms, two error evaluation criteria are used in this paper, which are the mean absolute error and the RMS error. The error functions are defined as follows:

$$
\begin{aligned}
E_{\text {mean }} & =\frac{\sum_{i=1}^{n}\left|\mathrm{TR}_{i}-\mathrm{TM}_{i}\right|}{n}, \\
E_{\mathrm{rms}} & =\frac{\sqrt{1 / n \sum_{i=1}^{n}\left(\mathrm{TR}_{i}-\mathrm{TM}_{i}\right)^{2}}}{\mathrm{TM}_{\text {mean }}} \times 100 \%,
\end{aligned}
$$

where $n$ is the number of reconstructed temperature values, $\mathrm{TR}_{i}$ and $\mathrm{TM}_{i}$ represent the corresponding temperature values in the same coordinate between the reconstruction results and the simulated temperature field model provided by Dongfang Boiler Works, and $\mathrm{TM}_{\text {mean }}$ represents the average temperature of the simulated temperature field model.

In order to comprehensively analyze the reconstruction effect of the temperature field, in addition to the global analysis of the reconstruction error, the reconstruction error is analyzed and compared from the central area and the edge area, where the central area refers to the part that can be 


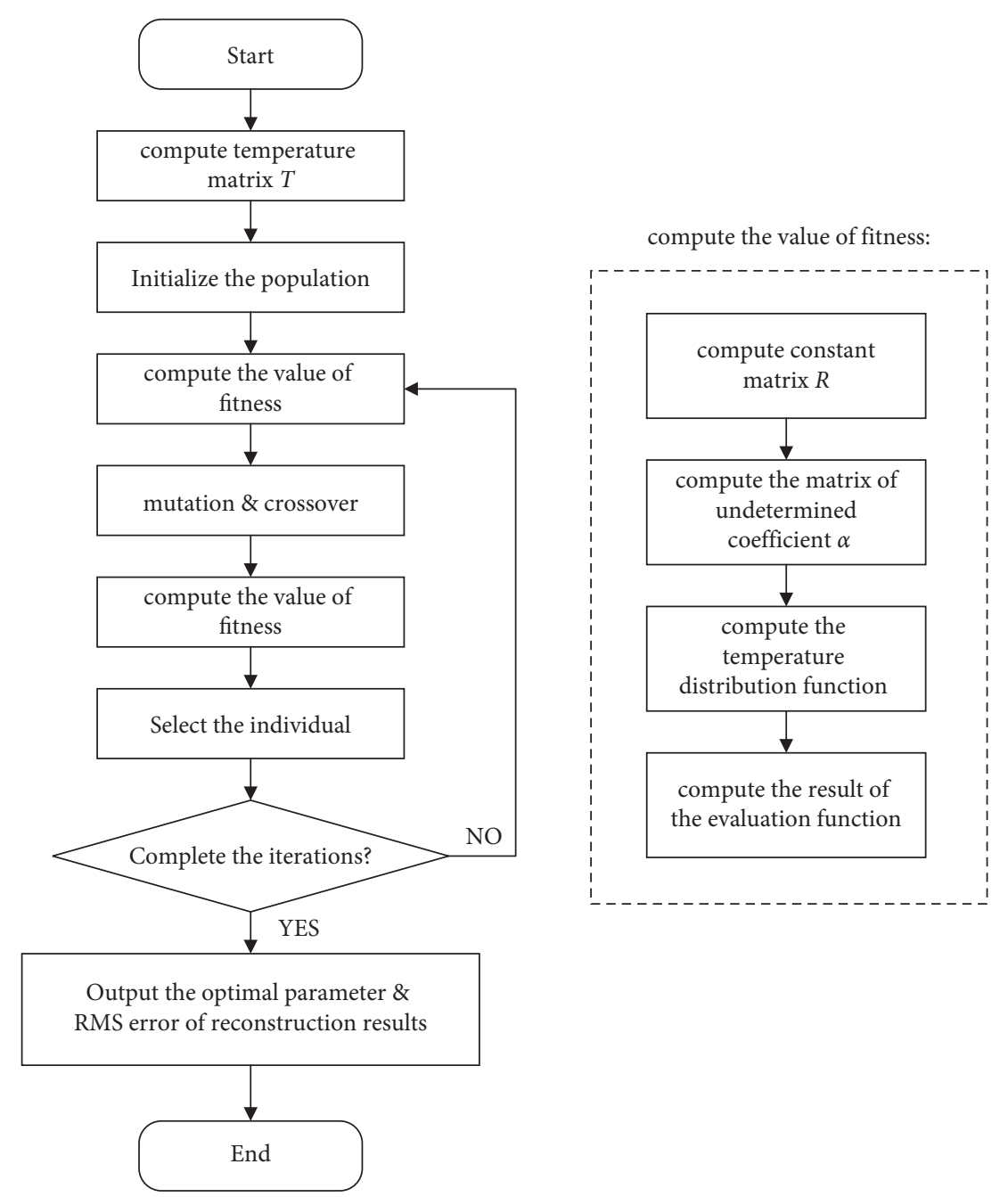

FIGURE 2: Work flow of the method for determining the optimal parameter of RBF.

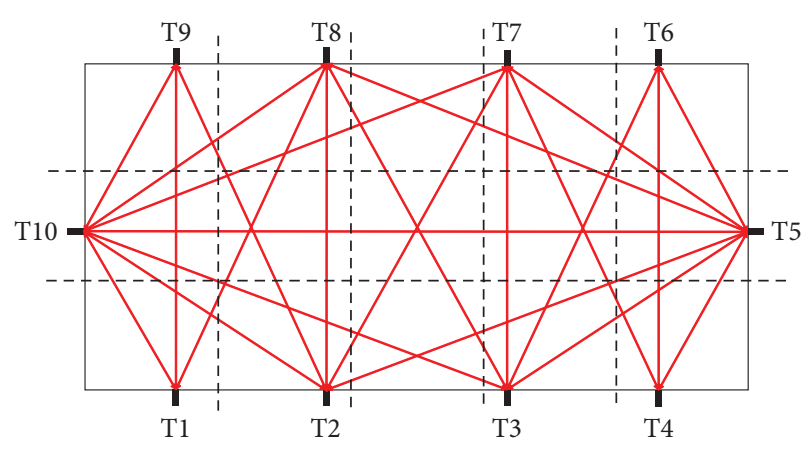

FIGURE 3: Transducer arrangement and division of the measured region.

reconstructed by LSM-BI, and the edge area refers to the part of the temperature field to be measured except the central area. For LSM-BI, error in the central area is considered global error due to the absence of edge temperature information. Global error, error in the central area, and error in the edge area of the three temperature field reconstruction results are shown in Tables 1-3.
From Figures 4-6, it can be seen that the EF-RBFI algorithm can effectively reconstruct the temperature field without the loss of temperature information in the edge area. Compared with other algorithms, the reconstructed results are closer to the temperature field model. Especially for the reconstruction of the edge area, LSM-BI has the problem of temperature loss at the edge. The reconstruction results of LSM-SP and ART-SP have a large fluctuation in the temperature of the edge area. LSM-KR does not show the decreasing trend of the edge temperature very well. Therefore, the EF-RBFI algorithm provides a better reconstruction effect.

Table 1 shows that, for a single-peak temperature field, the mean absolute error and RMS error of the edge area of the reconstruction result of the EF-RBFI algorithm are smaller than other comparable reconstruction algorithms, which improves the reconstruction accuracy of the edge area. From the point of view of the global error, since the reconstruction result of the LSM-BI has a lack of edge temperature, the comparison of the global error of LSM-BI is not performed. Compared with other comparable algorithms, the EF-RBFI algorithm has the smallest global error and the best reconstruction effect. It can be seen from Table 2 that, for the double-peak temperature field, the 


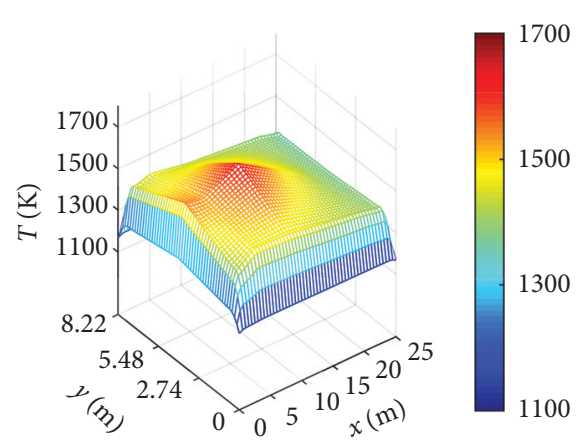

(a)

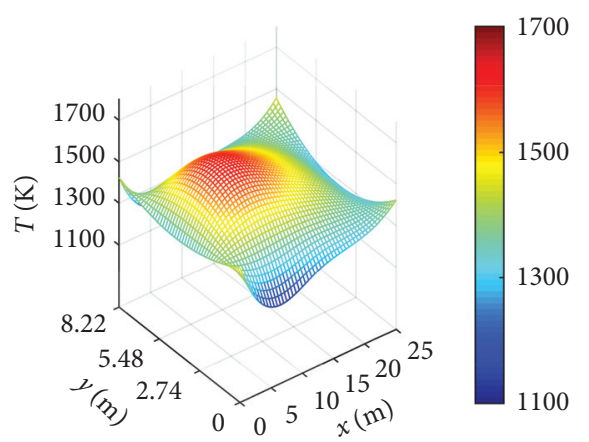

(c)

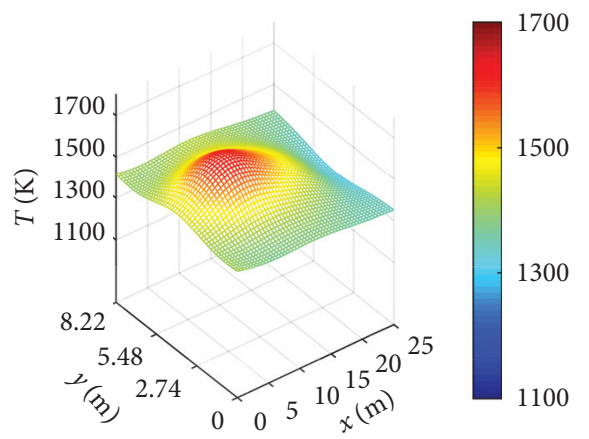

(e)

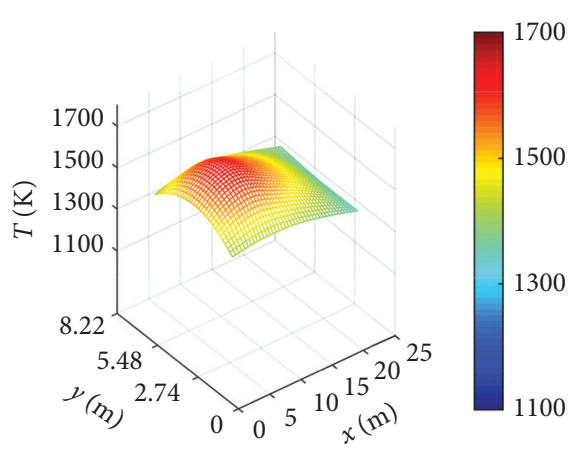

(b)

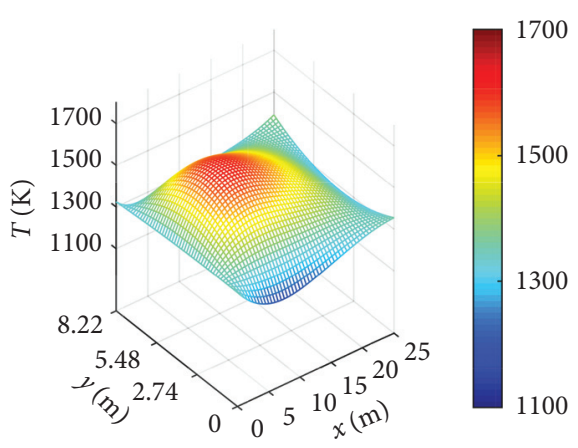

(d)

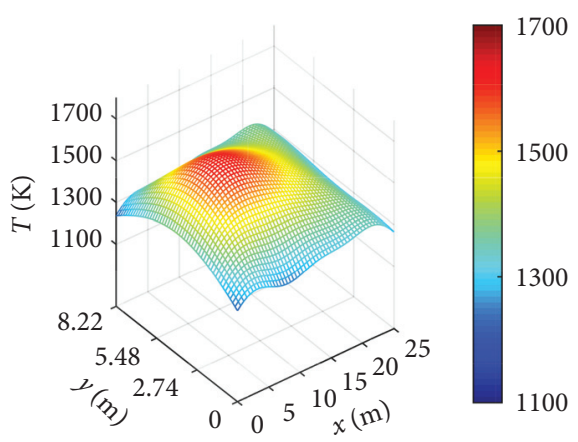

(f)

FIgURe 4: Reconstruction results for the single-peak temperature field. (a) Model. (b) LSM-BI. (c) LSM-SP. (d) ART-SP. (e) LSM-KR. (f) EF-RBFI.

reconstruction error of the LSM-SP and the ART-SP are relatively large. From the perspective of global error, the RMS error of the reconstruction result of the EF-RBFI algorithm is reduced by $2.2 \%$ compared with the minimum RMS error in other comparable algorithms. Table 3 indicates that, for the high-low peak temperature field, the EF-RBFI algorithm has the highest reconstruction accuracy compared to other algorithms from a global perspective. It can be seen from the quantitative analysis in Tables 1-3 that, compared with other comparable algorithms, the EF-RBFI algorithm has higher reconstruction quality for both the central area and the edge area, and the EF-RBFI algorithm achieves high-precision temperature field reconstruction.

4.3. The Effect of Noise on Reconstruction Results. Due to the deviation of TOF in the actual measurement, in order to simulate the actual measured value of TOF, different levels of noise are added to the theoretical value of TOF, as follows:

$$
t_{m}=t_{a} \times\left(1+\alpha \times N_{\text {noise }}\right),
$$

where $t_{\mathrm{m}}$ represents the TOF measurement data of the ultrasound, $t_{\mathrm{a}}$ is the actual TOF data of the ultrasound, $\alpha$ represents the noise level, and $N_{\text {noise }}$ is the Gaussian noise of the standard normal distribution. Table 4 shows the reconstruction error of the EF-RBFI algorithm under the noise levels of $1 \%, 3 \%$, and $5 \%$, respectively.

Table 4 reflects the robustness of the EF-RBFI algorithm. Although noise is added, the reconstruction error is still within an acceptable range, and the reconstruction result is not extremely deteriorated due to the added noise. At the same time, it can be seen that, as the noise level increases, the mean absolute error and RMS error of the temperature field reconstruction results are also gradually increasing. Therefore, the measurement accuracy of TOF directly affects the reconstruction effect of the temperature field. The higher the accuracy is, the better the reconstruction effect is. The results 


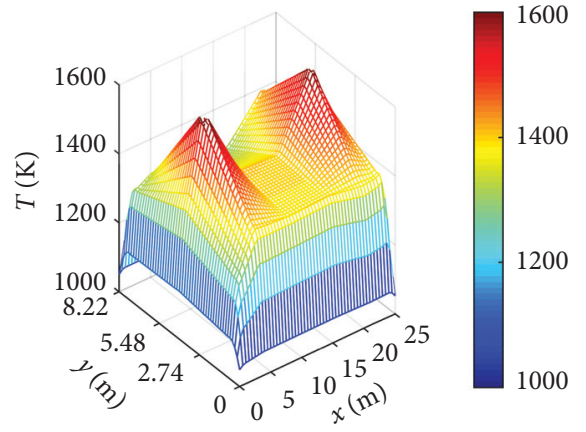

(a)

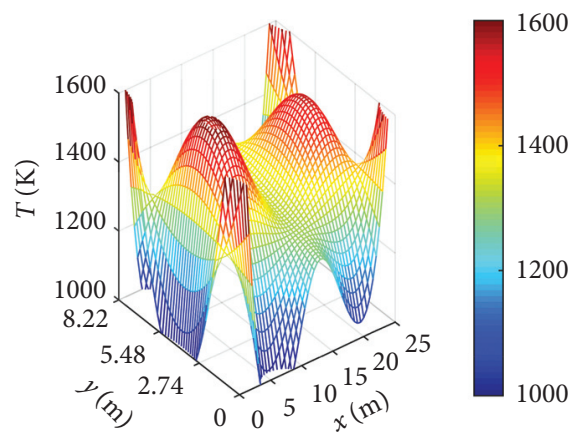

(c)

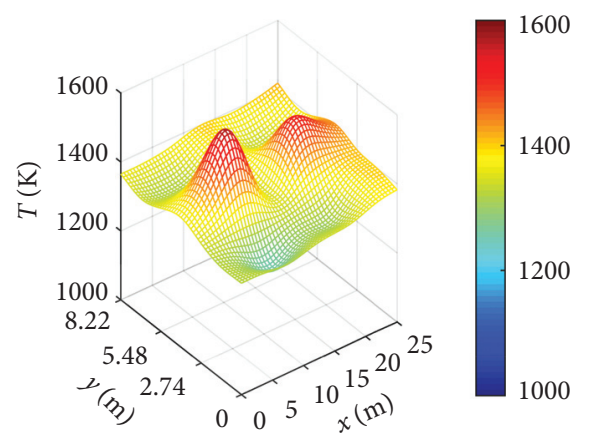

(e)

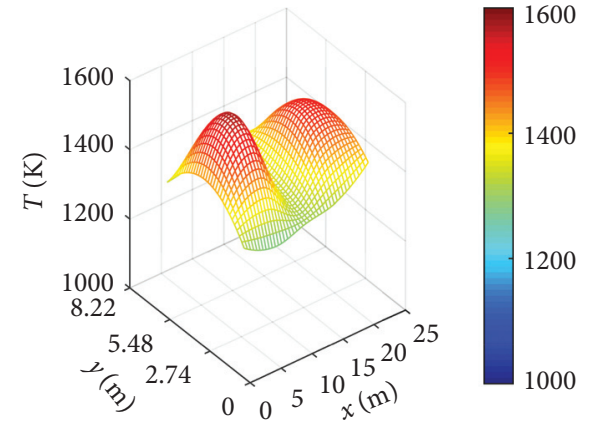

(b)

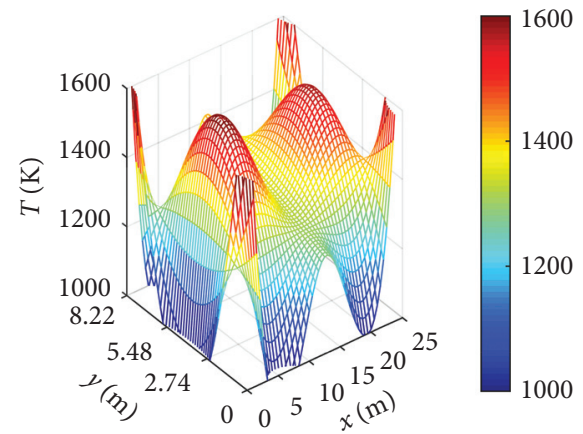

(d)

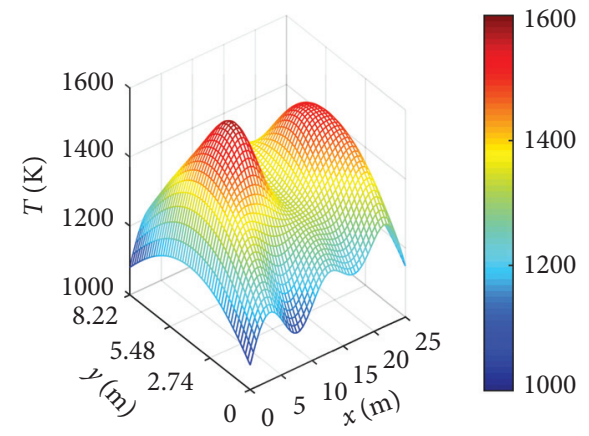

(f)

Figure 5: Reconstruction results for the double-peak temperature field. (a) Model. (b) LSM-BI. (c) LSM-SP. (d) ART-SP. (e) LSM-KR. (f) EF-RBFI.

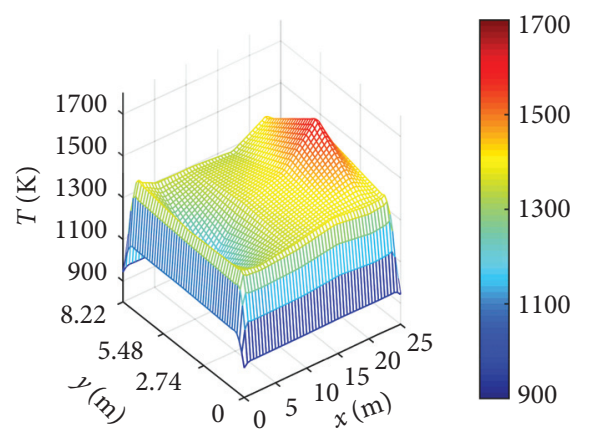

(a)

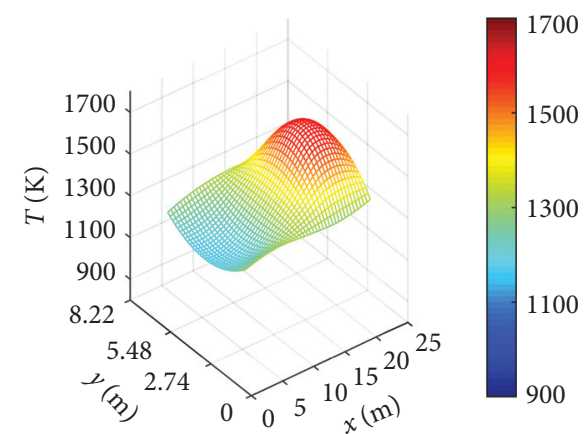

(b)

Figure 6: Continued. 


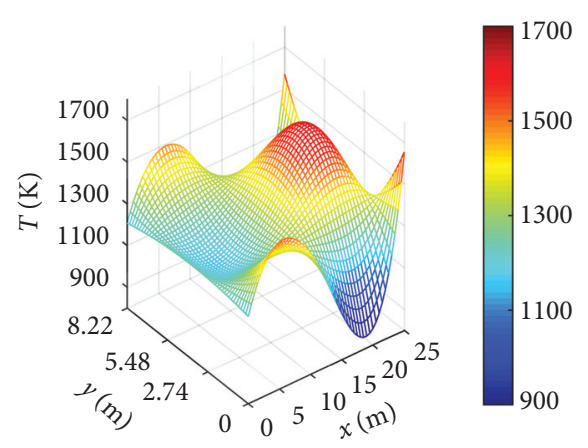

(c)

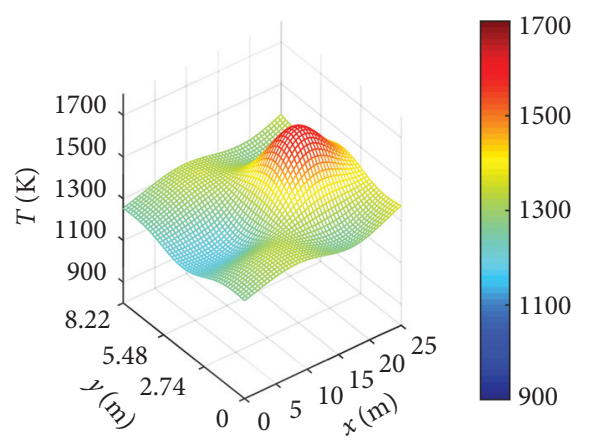

(e)

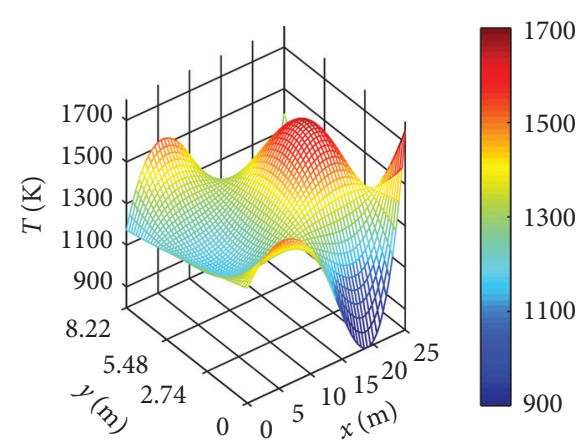

(d)

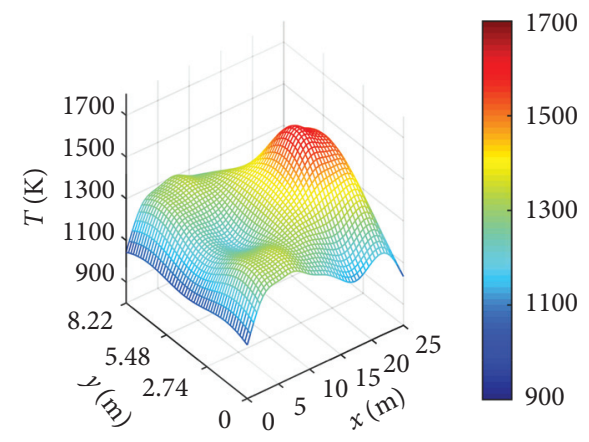

(f)

FIgURE 6: Reconstruction results for the high-low peak temperature field. (a) Model. (b) LSM-BI. (c) LSM-SP. (d) ART-SP. (e) LSM-KR. (f) EF-RBFI.

TABLE 1: Single-peak temperature field reconstruction error.

\begin{tabular}{lccccc}
\hline \multirow{2}{*}{ Reconstruction method } & \multicolumn{2}{c}{ Error in the central area } & \multicolumn{2}{c}{ Error in the edge area } & \multicolumn{2}{c}{ Global error } \\
& $E_{\text {mean }}(\mathrm{K})$ & $E_{\text {rms }}(\%)$ & $E_{\text {mean }}(\mathrm{K})$ & $E_{\text {rms }}(\%)$ & $E_{\text {mean }}(\mathrm{K})$ \\
\hline LSM-BI & 20.11 & 1.71 & 1 & 1 & 20.11 \\
LSM-SP & 20.75 & 1.78 & 67.98 & 6.03 & 43.41 \\
ART-SP & 19.03 & 1.61 & 66.29 & 5.58 & 41.71 \\
LSM-KR & 21.60 & 1.90 & 63.26 & 6.21 & 41.59 \\
EF-RBFI & 19.56 & 1.70 & 61.36 & 5.22 & 3.93 \\
\hline
\end{tabular}

TABLe 2: Double-peak temperature field reconstruction error.

\begin{tabular}{lccccc}
\hline \multirow{2}{*}{ Reconstruction method } & \multicolumn{2}{c}{ Error in the central area } & \multicolumn{2}{c}{ Error in the edge area } & \multicolumn{2}{c}{ Global error } \\
& $E_{\text {mean }}(\mathrm{K})$ & $E_{\text {rms }}(\%)$ & $E_{\text {mean }}(\mathrm{K})$ & $E_{\text {rms }}(\%)$ & $E_{\text {mean }}(\mathrm{K})$ \\
\hline LSM-BI & 52.23 & 4.24 & 1 & 1 & 52.23 \\
LSM-SP & 47.27 & 3.89 & 141.50 & 14.83 & 92.48 \\
ART-SP & 50.89 & 4.09 & 147.41 & 14.60 & 9.24 \\
LSM-KR & 62.95 & 5.15 & 99.22 & 10.17 & 10.19 \\
EF-RBFI & 47.95 & 3.92 & 77.58 & 7.11 & 80.35 \\
\hline
\end{tabular}

TABle 3: High-low peak temperature field reconstruction error.

\begin{tabular}{|c|c|c|c|c|c|c|}
\hline \multirow{2}{*}{ Reconstruction method } & \multicolumn{2}{|c|}{ Error in the central area } & \multicolumn{2}{|c|}{ Error in the edge area } & \multicolumn{2}{|c|}{ Global error } \\
\hline & $E_{\text {mean }}(\mathrm{K})$ & $E_{\mathrm{rms}}(\%)$ & $E_{\text {mean }}(\mathrm{K})$ & $E_{\mathrm{rms}}(\%)$ & $E_{\text {mean }}(\mathrm{K})$ & $E_{\mathrm{rms}}(\%)$ \\
\hline LSM-BI & 49.32 & 4.34 & 1 & 1 & 49.32 & 4.34 \\
\hline LSM-SP & 50.21 & 4.40 & 129.14 & 13.46 & 88.08 & 9.56 \\
\hline ART-SP & 53.19 & 4.71 & 136.31 & 14.17 & 93.07 & 10.09 \\
\hline LSM-KR & 50.14 & 4.51 & 102.81 & 11.11 & 75.41 & 8.14 \\
\hline EF-RBFI & 42.23 & 3.76 & 103.01 & 9.92 & 71.39 & 7.19 \\
\hline
\end{tabular}


TABLE 4: Reconstruction error of the EF-RBFI algorithm with noise.

\begin{tabular}{|c|c|c|c|c|c|c|c|}
\hline \multirow{2}{*}{$\alpha(\%)$} & \multirow{2}{*}{ Type of temperature field } & \multicolumn{2}{|c|}{ Error in the central area } & \multicolumn{2}{|c|}{ Error in the edge area } & \multicolumn{2}{|c|}{ Global error } \\
\hline & & $E_{\text {mean }}(\mathrm{K})$ & $E_{\mathrm{rms}}(\%)$ & $E_{\text {mean }}(\mathrm{K})$ & $E_{\mathrm{rms}}(\%)$ & $E_{\text {mean }}(\mathrm{K})$ & $E_{\mathrm{rms}}(\%)$ \\
\hline \multirow{3}{*}{1} & Single-peak & 24.59 & 2.05 & 69.17 & 5.67 & 45.98 & 4.08 \\
\hline & Double-peak & 51.66 & 4.22 & 82.91 & 7.47 & 66.65 & 5.86 \\
\hline & High-low peak & 47.03 & 4.08 & 108.94 & 10.27 & 76.74 & 7.50 \\
\hline \multirow{3}{*}{3} & Single-peak & 49.25 & 3.67 & 86.34 & 6.89 & 67.05 & 5.35 \\
\hline & Double-peak & 64.88 & 5.38 & 94.98 & 8.43 & 79.32 & 6.88 \\
\hline & High-low peak & 62.64 & 5.28 & 122.47 & 11.13 & 91.35 & 8.41 \\
\hline \multirow{3}{*}{5} & Single-peak & 79.49 & 5.57 & 104.23 & 8.35 & 91.36 & 6.94 \\
\hline & Double-peak & 85.60 & 6.92 & 109.07 & 9.65 & 96.86 & 8.22 \\
\hline & High-low peak & 83.76 & 6.85 & 137.14 & 12.15 & 109.37 & 9.58 \\
\hline
\end{tabular}

show that the noise interference should be reduced and the measurement quality of TOF data should be further improved.

\section{Conclusions and Future Research}

Furnace temperature distribution is an important parameter to understand and optimize combustion state, and AT is a noninvasive method to measure the combustion temperature field. In order to achieve high-quality temperature field reconstruction based on $\mathrm{AT}$, a temperature field reconstruction algorithm based on the radial basis function (RBF) interpolation method optimized by the evaluation function is proposed. We studied the relationship between the parameter of RBF and RMS error of reconstruction results and proposed a determination method for the optimal parameter of RBF based on the evaluation function which employs the DE algorithm to search for the optimal parameter. Through the reconstruction experiment of three temperature fields provided by Dongfang Boiler Works, it is proved that the EF-RBFI algorithm overcomes the problem of the lack of edge temperature information and can realize the global reconstruction of the temperature field, which proves the effectiveness of the algorithm. The error analysis of the experiment shows that the EF-RBFI algorithm has good reconstruction performance regardless of the temperature field reconstruction in the central area or the edge area. Therefore, the EF-RBFI algorithm can effectively describe the temperature field information and achieve high-precision reconstruction. In reality, considering that the noise level will affect the error of the temperature field reconstruction results, the de-noising of ultrasonic TOF data will be the direction of further research in the future.

\section{Data Availability}

The temperature field model data used to support the findings of this study are included within the supplementary information files.

\section{Conflicts of Interest}

The authors declare that there are no conflicts of interest regarding the publication of this paper.

\section{Acknowledgments}

This work was supported by the Major State Basic Research Development Program (973 Program Grant no. 2013CB328903-2).

\section{Supplementary Materials}

The original temperature value of each temperature field is as follows: (1) temperature value of single-peak temperature field (K); (2) temperature value of double-peak temperature field $(\mathrm{K})$; (3) temperature value of high-low peak temperature field (K). (Supplementary Materials)

\section{References}

[1] S. P. Zhang, G. Q. Shen, L. S. An et al., "Online monitoring of the two-dimensional temperature field in a boiler furnace based on acoustic computed tomography," Applied Thermal Engineering, vol. 75, pp. 958-966, 2015.

[2] O. Hwang, M. C. Lee, W. B. Weng et al., "Development of novel ultrasonic temperature measurement technology for combustion gas as a potential indicator of combustion instability diagnostics," Applied Thermal Engineering, vol. 159, Article ID 113905, 2019.

[3] Q. Kong, G. S. Jiang, Y. S. Liu et al., "3D temperature distribution reconstruction in furnace based on acoustic tomography," Mathematical Problems in Engineering, vol. 2019, Article ID 1830965, 15 pages, 2019.

[4] S. P. Zhang, G. Q. Shen, L. S. An et al., "Monitoring ash fouling in power station boiler furnaces using acoustic pyrometry," Chemical Engineering Science, vol. 126, pp. 216-223, 2015.

[5] M. Bramanti, E. A. Salerno, A. Tonazzini et al., "An acoustic pyrometer system for tomographic thermal imaging in power plant boilers," IEEE Transactions on Instrumentation and Measurement, vol. 45, no. 1, pp. 159-161, 1996.

[6] W. Y. Tsai, C. F. Huang, and T. L. Liao, "New implementation of high-precision and instant-response air thermometer by ultrasonic sensors," Sensors and Actuators A: Physical, vol. 117, no. 1, pp. 88-94, 2005.

[7] W. Y. Tsai, H. C. Chen, and T. L. Liao, "High accuracy ultrasonic air temperature measurement using multi-frequency continuous wave," Sensors and Actuators A: Physical, vol. 132, no. 2, pp. 526-532, 2006.

[8] Y. Liu, S. Liu, J. Lei et al., "A method for simultaneous reconstruction of temperature and concentration distribution 
in gas mixtures based on acoustic tomography," Acoustical Physics, vol. 61, no. 5, pp. 597-605, 2015.

[9] H. Yan, G. N. Chen, Y. G. Zhou et al., "Primary study of temperature distribution measurement in stored grain based on acoustic tomography," Experimental Thermal and Fluid Science, vol. 42, pp. 55-63, 2012.

[10] S. Alzebda and A. N. Kalashnikov, "Ultrasonic sensing of temperature of liquids using inexpensive narrowband piezoelectric transducers," IEEE Transactions on Ultrasonics, Ferroelectrics, and Frequency Control, vol. 57, no. 12, pp. 2704-2711, 2010.

[11] W. Fan, C. A. Chen, and Y. Chen, "Calibration of an acoustic system for measuring 2-D temperature distribution around hydrothermal vents," Ultrasonics, vol. 53, no. 4, pp. 897-906, 2013.

[12] W. Fan, Y. Chen, H. C. Pan et al., "Experimental study on underwater acoustic imaging of 2-D temperature distribution around hot springs on floor of Lake Qiezishan, China," Experimental Thermal and Fluid Science, vol. 34, no. 8, pp. 1334-1345, 2010.

[13] K. J. Dharmaraj, C. D. Cox, A. M. Strauss et al., "Ultrasonic thermometry for friction stir spot welding," Measurement, vol. 49, pp. 226-235, 2014.

[14] C. Hopmann, J. Wipperfurth, and M. Schongart, "Spatially resolved temperature measurement in injection moulding using ultrasound tomography," in Proceedings of 32nd International Conference of the Polymer-Processing-Society (PPS), Lyon, France, July 2016.

[15] D. Wei, S. You-An, S. Bi-Nan et al., "Reconstruction of internal temperature distributions in heat materials by ultrasonic measurements," Applied Thermal Engineering, vol. 112, pp. 38-44, 2017.

[16] Y. L. Jia, M. Puga, A. E. Butterfield et al., "Ultrasound measurements of temperature profile Across gasifier refractories: method and initial validation," Energy \& Fuels, vol. 27, no. 8, pp. 4270-4277, 2013.

[17] L. B. Xie, T. Liu, Z. G. Zhang et al., "The 2-D temperature reconstruction based on the algorithm of interpolation and iterative," Proceedings of the Chinese Society for Electrical Engineering, vol. 24, no. 10, pp. 249-252, 2004.

[18] S. Liu, S. Liu, and T. Ren, "Acoustic tomography reconstruction method for the temperature distribution measurement," IEEE Transactions on Instrumentation and Measurement, vol. 66, no. 8, pp. 1936-1945, 2017.

[19] A. H. Andersen and A. C. Kak, "Simultaneous algebraic reconstruction technique (SART): a superior implementation of the ART algorithm," Ultrasonic Imaging, vol. 6, no. 3, pp. 81-94, 1984.

[20] R. Wang, L. L. An, G. Q. Shen et al., "Acoustic reconstruction of three-dimensional temperature field based on regularized SVD algorithm," Chinese Journal of Computational Physics, vol. 32, no. 2, pp. 195-201, 2015.

[21] F. Tian, Z. S. Liu, L. Q. Liu et al., "Simulation research on reconstruction algorithm of complex temperature field based on RBF neural network," in Proceedings of 6th International Conference on Intelligent Systems Design and Applications, pp. 93-97, ISDA, Jinan, China, October 2006.

[22] X. Zhou, Q. Wang, Z. N. Miao, and W. G. Wu, “Three-dimensional temperature field reconstruction algorithm based on RBF neural network," Instrument Technique and Sensor, vol. 5, pp. 99-102, 2013.

[23] J. Lu, K. Wakai, S. Takahashi et al., "Acoustic computer tomographic pyrometry for two-dimensional measurement of gases taking into account the effect of refraction of sound wave paths," Measurement Science and Technology, vol. 11, no. 6, pp. 692-697, 2000.

[24] H. Yan, X. Lin, and S. H. Wang, "Reconstruction of threedimensional temperature field based on least-square method and Kriging interpolation," Journal of Shenyang University of Technology, vol. 36, no. 3, pp. 303-307, 2014.

[25] X. H. Shen, Q. Y. Xiong, W. R. Shi et al., “A new algorithm for reconstructing two-dimensional temperature distribution by ultrasonic thermometry," Mathematical Problems in Engineering, vol. 2015, Article ID 916741, 10 pages, 2015.

[26] J. G. Wang and G. R. Liu, "On the optimal shape parameters of radial basis functions used for 2-D meshless methods," Computer Methods in Applied Mechanics and Engineering, vol. 191, pp. 2611-2630, 2002.

[27] G. E. Fasshatier and J. G. Zhang, "On choosing "optimal" shape parameters for RBF approximation," Numerical Algorithms, vol. 45, pp. 345-368, 2007.

[28] H. T. Cheng and Z. G. Liu, "Improved Differential Evolution with Parameter Adaption Based on Population Diversity," in Proceedings of 3rd IEEE International Conference On Image, Vision And Computing (ICIVC), pp. 901-905, Chongqing, China, June 2018.

[29] L. Wang, L. Peng, D. Xia et al., "BP neural network incorporating self-adaptive differential evolution algorithm for time series forecasting," Computer Engineering and Science, vol. 37, no. 12, pp. 2270-2275, 2015.

[30] S. Das, S. S. Mullick, and P. N. Suganthan, "Recent advances in differential evolution - An updated survey," Swarm and Evolutionary Computation, vol. 27, pp. 1-30, 2016. 